\title{
Association between sodium glucose co-transporter 2 inhibitors and a reduced risk of heart failure in patients with type 2 diabetes mellitus: a real-world nationwide population-based cohort study
}

\author{
Young-Gun Kim ${ }^{1,2}$, Seung Jin $\mathrm{Han}^{3}$, Dae Jung Kim³ ${ }^{3}$ Kwan-Woo Lee ${ }^{3}$ and Hae Jin Kim ${ }^{3 *}$ (D
}

\begin{abstract}
Background: Recently, two large randomized controlled trials which only included patients with underlying cardiovascular disease (CVD) or patients at high risk for CVD showed that two sodium glucose co-transporter 2 inhibitors (SGLT-2is) significantly reduced hospitalization for heart failure (hHF), with an early separation in the survival curves for hHF. There were concerns whether SGLT-2i use could protect hHF in patients without CVD and how soon SGLT2i-treated patients show a lower risk of hHF. Thus, we aimed to evaluate whether the heart failure protective effect of SGLT-2i differs depending on the underlying CVD and the prescription period compared with dipeptidyl peptidase-4 inhibitors (DPP-4i).
\end{abstract}

Methods: We performed a nationwide retrospective observational study to estimate the effect of SGLT-2i on HF. The 59,479 SGLT-2i new-users were matched with same number of DPP-4i new-users through propensity score matching using 53 confounding variables. Kaplan-Meier (K-M) curves and Cox proportional hazards regression analyses were used to estimate the risk of hospitalization for hHF.

Results: The incidence rates of hHF were 0.83 and 1.13 per 100 person-years in SGLT-2i-treated patients and DPP4i-treated patients, respectively. The hazard ratios of hHF were 0.66 (95\% confidence interval 0.58-0.75) in SGLT2i-treated patients compared with the DPP-4i-treated patients. Among the patients with underlying CVD, SGLT-2itreated patients were associated with a lower risk of hHF from 30 days to 3 years after initiating drugs compared with DPP-4i. However, SGLT-2i use only showed a lower risk of hHF with a significant difference 3 years after drug initiation among patients without underlying CVD.

Conclusions: Our findings suggest that SGLT-2i reduced hHF compared with DPP-4i. A heart failure protective effect of SGLT-2i use vs. DPP-4i use was shown 30 days after initiating the SGLT-2i among patients with established CVD, but this effect appeared later in patients without established CVD.

Keywords: Heart failure, Type 2 diabetes mellitus, Sodium glucose co-transporter 2 inhibitors dipeptidyl peptidase-4 inhibitor

\footnotetext{
*Correspondence: jinkim@ajou.ac.kr

${ }^{3}$ Department of Endocrinology and Metabolism, Ajou University School

of Medicine, Suwon, Republic of Korea

Full list of author information is available at the end of the article
} 


\section{Introduction}

Heart failure (HF) occurs in 1-2\% of the world's population [1]. Despite standard therapy, such as renin-angiotensin-aldosterone system inhibitors, beta blockers, or diuretics, there is a requirement for an unmet need [2]. In particular, HF is a major comorbidity in patients with type 2 diabetes mellitus (T2DM), which is caused by diverse pathogenic factors such as the cardiotoxic tetrad of coronary artery disease, diabetic cardiomyopathy, or hypertension. HF reduces quality of life, results in hospitalization, and contributes to a large socioeconomic loss $[1,3]$.

Some studies have indicated that HF is associated with oral hypoglycemic agents, which has attracted worldwide attention. Rosiglitazone has been withdrawn from the market because it was reported to cause HF. Saxagliptin has been reported to be associate with HF, which has caused global controversy $[4,5]$. Unlike these two drugs, empagliflozin reduced hospitalization for heart failure (hHF) by $35 \%$ in a randomized controlled trial (RCT) called the Empagliflozin Cardiovascular Outcome Event Trial in Type 2 Diabetes Mellitus Patients (EMPA-REG OUTCOME) [6], and this tendency was the same when only Asian patients were analyzed [7]. In another RCT, the Canagliflozin Cardiovascular Assessment Study (CANVAS) Program, canagliflozin reduced hHF, suggesting the protective effect of sodium glucose co-transporter 2 inhibitors (SGLT-2i) as a class effect on HF [8]. Although there is no RCT, multinational observational studies have shown that dapagliflozin is also protective against HF [9]. In the EMPA-REG OUTCOME trial, only patients with established cardiovascular disease (CVD) were included. About $70 \%$ of the participants in the CANVAS program had established CVD, and the remaining participants were patients with two or more CVD risks. Thus, after the results of these studies were published, concerns about the effect of SGLT-2i on HF in patients without established CVD were raised.

An early separation of survival curves for hHF was observed in the EMPA-REG OUTCOME trial and CANVAS Program, although no differences were observed in myocardial infarction or cerebral infarction $[6,8]$. This finding suggests that the HF protective effect of SGLT-2i could be derived through a non-glycemic anti-atherosclerotic mechanism, such as a diuretic effect, a hemo-concentration effect, a ketone body-producing effect, or a uric acid lowering effect. However, to the best of our knowledge, no observational study has been conducted to determine how soon SGLT-2i-treated patients show a lower incidence of hHF.

We compared HF risk of SGLT-2i with dipeptidyl peptidase-4 inhibitors (DPP-4i) as an active comparator because both drugs are similarly used as a second-line treatment and have a low risk of hypoglycemia and weight gain, as well as a potent glucose-lowering effect. Although there is still debate and no consensus, DPP-4i has been shown not to elevate the risk of heart failure in many studies [10-13].

We aimed to evaluate the HF protective effect of SGLT$2 \mathrm{i}$ compared with DPP-4i. In addition, we sought to estimate whether the HF protective effect of SGLT-2i differs depending on the underlying $C V D$ and the prescription period.

\section{Methods \\ Study design and data source}

We performed a nationwide retrospective observational study to estimate the effect of SGLT-2i on HF. The health records from the Korean Health Insurance Review and Assessment Service (HIRA) database were analyzed. All records were de-identified according to relevant laws and regulations. This database covers $>99 \%$ of the South Korean population and includes all health records, such as demographics, diagnoses (coded with International Classification of Diseases [ICD]-10), drug prescriptions, and procedures. We used the data from January 1, 2013 to June 30, 2017. Our study protocol was reviewed and approved by the Institutional Review Board (IRB) of Ajou University Hospital (AJIRB-MED-EXP-17-497), and informed consent was waived by the IRB.

\section{Patient cohort}

Patients aged $>19$ years with T2DM (ICD-10 code: E11) who were new users of SGLT-2i or DPP-4i were included in the cohort. A new user was defined as a patient who had more than a 1-year wash-out period before the first SGLT-2i or DPP-4i prescription (The first index date was Jan 1, 2014). The first prescribed drug was defined as the index drug and the first prescription date was designated the index date. Patients who were diagnosed with type 1 diabetes mellitus, end-stage renal disease, acquired immune deficiency syndrome, any malignancy, or previous hHF 60 days before the index date were excluded. A flow chart is shown in Fig. 1.

\section{Outcomes}

The study outcome was hHF (diagnosed as ICD-10 code I50 during the admission) after initiating SGLT-2i. The study cohort was stratified according to whether the patient had established CVD (diagnosed as HF, myocardial infarction, other ischemic heart disease, stroke, cerebrovascular disease, peripheral artery occlusive disease with an ICD-10 code, or received percutaneous coronary intervention or a coronary artery bypass graft). To evaluate whether the HF risk of SGLT-2i varied with the follow-up period after the time of initiation, analyses were 


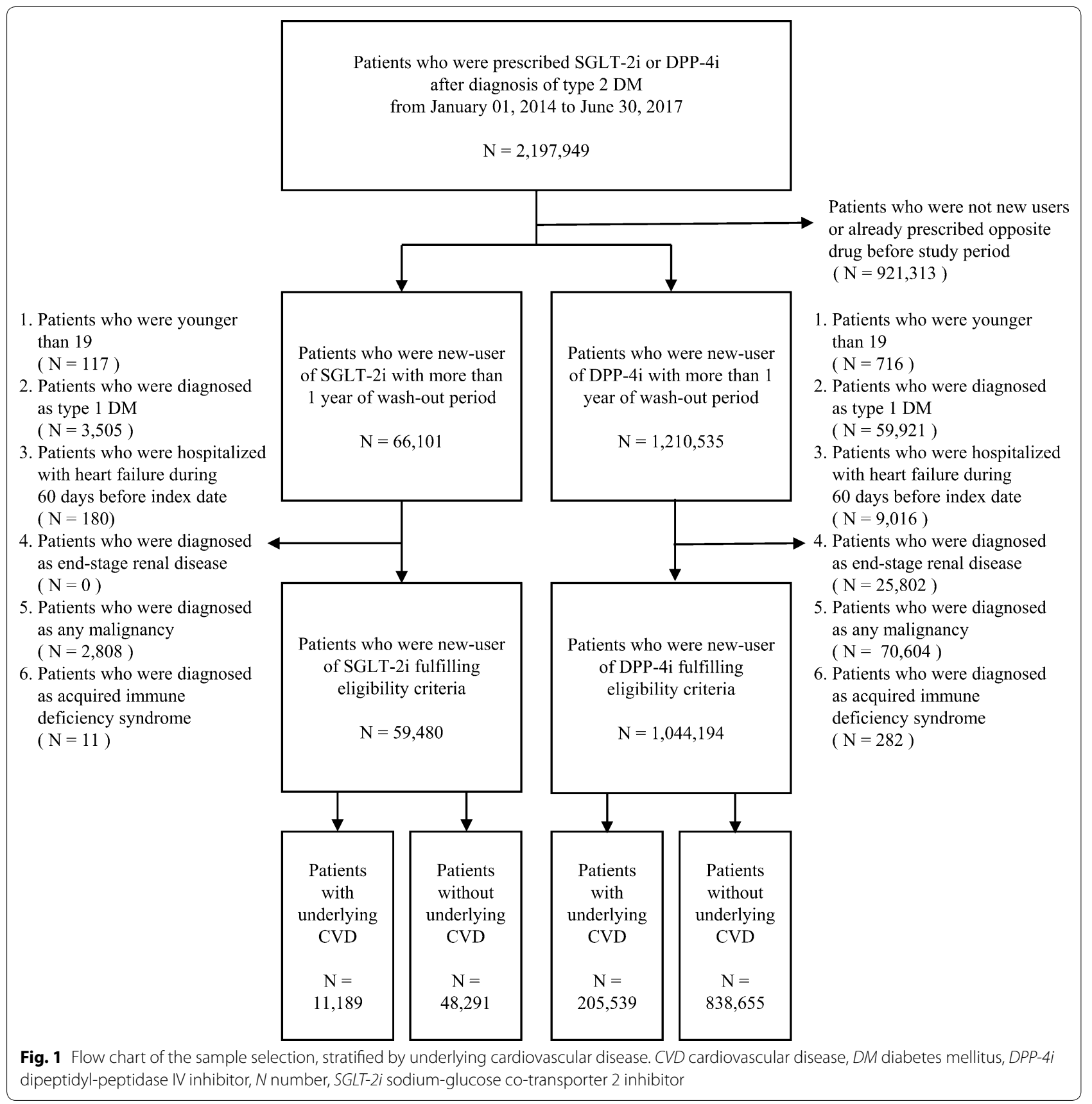

performed according to the time after initiation of the drug (30, 90, 180 days, 1 , and 3 years after the index date) in all patients and each CVD stratum.

\section{Statistical analysis}

All analyses were performed with SAS (ver. 9.4; SAS Institute, Cary, NC, USA) and R software (ver. 3.4.1; R Development Core Team, Vienna, Austria). All values are presented as mean \pm standard deviation (SD). To minimize differences in the baseline characteristics between the SGLT-2i and DPP-4i groups, propensity score matching was performed with 53 variables which were presented in Table 1 (sex, age, underlying disease [1 year prior to the index date], prescribed drugs [180 days prior to the index date, particularly beta-blockers, angiotensin converting enzyme inhibitors, angiotensin receptor blockers, aldosterone antagonists, loop diuretics, and thiazides, which may affect hospitalization for HF, were also included], cardiologist visits [30 days prior to the index date], hospitalization [30 or 31-365 days prior to the 
Table 1 Baseline characteristics of matched pairs in all patients

\begin{tabular}{|c|c|c|c|}
\hline & DPP-4i $(n=59,479)$ & SGLT-2i $(n=59,479)$ & $\begin{array}{l}\text { Standardized } \\
\text { difference }\end{array}$ \\
\hline Age (years), mean (SD) & $53.4(12.4)$ & $53.2(11.9)$ & 0.012 \\
\hline Male & 54.75 & 54.86 & 0.002 \\
\hline Hypertension & 60.46 & 60.33 & 0.003 \\
\hline Dyslipidemia & 81.98 & 82.05 & 0.002 \\
\hline Chronic kidney disease & 7.74 & 7.55 & 0.007 \\
\hline \multicolumn{4}{|l|}{ Cardiovascular disease } \\
\hline AMI & 1.57 & 1.43 & 0.011 \\
\hline Other ischemic heart disease & 14.25 & 14.02 & 0.007 \\
\hline Heart failure & 3.39 & 3.41 & 0.001 \\
\hline Cerebral infarction & 3.82 & 3.71 & 0.006 \\
\hline Cerebrovascular event & 5.33 & 5.24 & 0.004 \\
\hline Peripheral artery occlusive disease & 0.66 & 0.61 & 0.006 \\
\hline \multicolumn{4}{|l|}{ Coronary revascularization procedures } \\
\hline Coronary artery bypass graft & 0.04 & 0.03 & 0.003 \\
\hline Percutaneous coronary intervention & 2.02 & 1.99 & 0.002 \\
\hline \multicolumn{4}{|l|}{ Microvascular complications of diabetes } \\
\hline Nephropathy & 10.46 & 10.34 & 0.004 \\
\hline Neuropathy & 7.17 & 6.99 & 0.007 \\
\hline Retinopathy & 13.04 & 12.93 & 0.003 \\
\hline Atrial fibrillation & 1.58 & 1.52 & 0.004 \\
\hline Other heart disease & 11.79 & 11.71 & 0.002 \\
\hline Hypoglycemia & 2.2 & 2.16 & 0.002 \\
\hline Asthma & 12.56 & 12.49 & 0.002 \\
\hline Chronic obstructive pulmonary disease & 4.84 & 4.74 & 0.005 \\
\hline Connective tissue disease & 3.57 & 3.58 & 0.001 \\
\hline Pancreatitis & 1.5 & 1.49 & 0.001 \\
\hline Osteoporosis & 7.67 & 7.36 & 0.012 \\
\hline Alcohol intake ${ }^{a}$ & 4.66 & 4.65 & $<0.001$ \\
\hline Smoking ${ }^{\mathrm{a}}$ & 0.15 & 0.16 & 0.002 \\
\hline Obesity $^{a}$ & 0.39 & 0.39 & 0.001 \\
\hline \multicolumn{4}{|l|}{ Medication use (180 days prior to index date) } \\
\hline \multicolumn{4}{|l|}{ Antidiabetic agent } \\
\hline Metformin & 80.88 & 81.18 & 0.008 \\
\hline Sulfonylurea & 37.29 & 36.77 & 0.011 \\
\hline Thiazolidinediones & 9.74 & 9.11 & 0.022 \\
\hline Alpha-glucosidase inhibitor & 4.86 & 4.71 & 0.007 \\
\hline Meglitinide & 0.81 & 0.73 & 0.009 \\
\hline Insulin & 14.45 & 13.84 & 0.017 \\
\hline \multicolumn{4}{|l|}{ Diuretics } \\
\hline Loop diuretics & 3.83 & 3.79 & 0.002 \\
\hline Thiazide & 14.86 & 14.68 & 0.005 \\
\hline Aldosterone antagonist & 2.01 & 1.92 & 0.006 \\
\hline Potassium sparing diuretics & 0.05 & 0.06 & 0.006 \\
\hline \multicolumn{4}{|l|}{ Anti-hypertensive agent } \\
\hline Calcium channel blocker & 29.7 & 29.74 & 0.001 \\
\hline ACEI & 3.06 & 2.95 & 0.007 \\
\hline ARB & 47.68 & 47.62 & 0.001 \\
\hline Beta blocker & 9.92 & 9.65 & 0.009 \\
\hline Alpha blocker & 0.71 & 0.67 & 0.005 \\
\hline
\end{tabular}


Table 1 (continued)

\begin{tabular}{llll}
\hline & DPP-4i $(\mathbf{n = 5 9 , 4 7 9 )}$ & SGLT-2i $(\mathbf{n = 5 9 , 4 7 9 )}$ & $\begin{array}{c}\text { Standardized } \\
\text { difference }\end{array}$ \\
\hline Digoxin & & 0.72 & 0.002 \\
Aspirin & 0.7 & 22.98 & 0.01 \\
P2Y12 inhibitor & 23.39 & 8.38 & 0.006 \\
Warfarin & 8.55 & 0.58 & 0.001 \\
NOAC & 0.58 & 0.53 & 0.017 \\
Lipid-lowering agent & 0.66 & & 0.008 \\
Statin & & 53.02 & 0.74 \\
$\quad$ Fibrate & 53.42 & 8.51 & 0.005 \\
$\quad$ Ezetimibe & 10.59 & 12.4 & 0.001 \\
Cardiologist visit (30 days prior to index date) & 8.54 & 6.9 & 0.002 \\
Hospitalization (30 days prior to index date) & 12.47 & 20.3 & 0.017 \\
Hospitalization (30-365 days prior to index date) & 7.33 & 5.84 & 0.016 \\
Emergency department visit (365 days prior to index date) & 20.96 & & 0.010 \\
\hline Data & 6.08 & &
\end{tabular}

Data presented as frequencies in percentage or means (SD)

Less than $0.1(10 \%)$ on the absolute value of standardized difference was considered as a negligible difference between groups. The mean (SD) standardized difference of all covariates was $0.59 \%(0.49 \%)$

$A C E l$ angiotensin-converting-enzyme inhibitor, $A M I$ acute myocardial infarction, $A R B$ angiotensin II receptor antagonists, $D P P-4 i$ dipeptidyl-peptidase IV inhibitor, NOAC novel oral anticoagulant, SD standard deviation, SGLT2i sodium-glucose co-transporter 2 inhibitor, SU sulfonylurea

a Confirmed by diagnosis code (International Classification of Diseases, 10th revision)

index date], emergency department visit [365 days prior to the index date]). The nearest neighbor matching was used with a caliper (0.1). Propensity score matching was performed three times (DPP-4i group vs. SGLT-2i group in all patients, and CVD stratification) with a 1:1 ratio. Differences between the two groups were calculated with standardized differences and absolute values $<0.1(10 \%)$ of standardized differences were considered to be no difference.

After propensity score matching, we performed survival analyses to estimate the effect of SGLT-2i on hHF. The Kaplan-Meier estimates were performed on matched pairs using 1 minus the Kaplan-Meier estimate. Cox proportional hazards regressions were performed with matched pairs in all patients and each CVD stratum. To determine whether the hHF risk of SGLT-2i varied with the follow-up period after the time of initiation, analyses were performed according to the time after initiating the drug $(30,90,180$ days, 1 , and 3 years after the index date).

\section{Results}

\section{Study population and patient characteristics}

A total of 1,103,674 new users were included in the cohort $(1,044,194$ new users in the DPP-4i group and 59,480 new users in the SGLT-2i group) and 1,128,528 person-years. In total, 59,479 pairs were included after propensity score matching. According to underlying
CVD, 11,188 and 48,290 pairs were matched in patients with and without underlying CVD, respectively.

The baseline characteristics of all patients before propensity score matching are presented in Additional file 1: Table $\mathrm{S} 1$ and those of the matched patients are presented in Table 1 and Additional file 1: Tables S2 and S3. The standardized differences in all variables were $<0.1(10 \%)$ and means (SD) of the standardized differences were $0.59 \%(0.49 \%), 1.01 \%(1.33 \%)$, and $0.96 \%(2.03 \%)$ in all patients and patients with and without CVD, respectively. Thus, the differences between matched pairs were statistically negligible. The mean follow-up period of the matched patients was 318.5 days. The SGLT2 inhibitor group consisted of dapagliflozin (90.2\%) and ipragliflozin (9.8\%) and the DPP-4 inhibitors group consisted of linagliptin (26.3\%), sitagliptin (26.1\%), gemigliptin (15.6\%), vildagliptin (10.0\%), alogliptin (7.8\%), saxagliptin (5.4\%), teneligliptin (5.2\%), anagliptin (2.0\%), and evogliptin (1.7\%).

\section{Heart failure risk of SGLT-2i vs. DPP-4i}

In total, $1025 \mathrm{hHF}$ events were observed among the matched patients during the follow-up period. The incidence rates of hHF were 0.83 and 1.13 per 100 personyears in SGLT-2i-treated patients and DPP-4i-treated patients, respectively. The hazard ratios of hHF were 0.66 (95\% confidence interval [CI] 0.58-0.75, p $<0.001$ ) in SGLT-2i-treated patients compared with the DPP4i-treated patients (Fig. 2 and Table 2). SGLT-2i use 


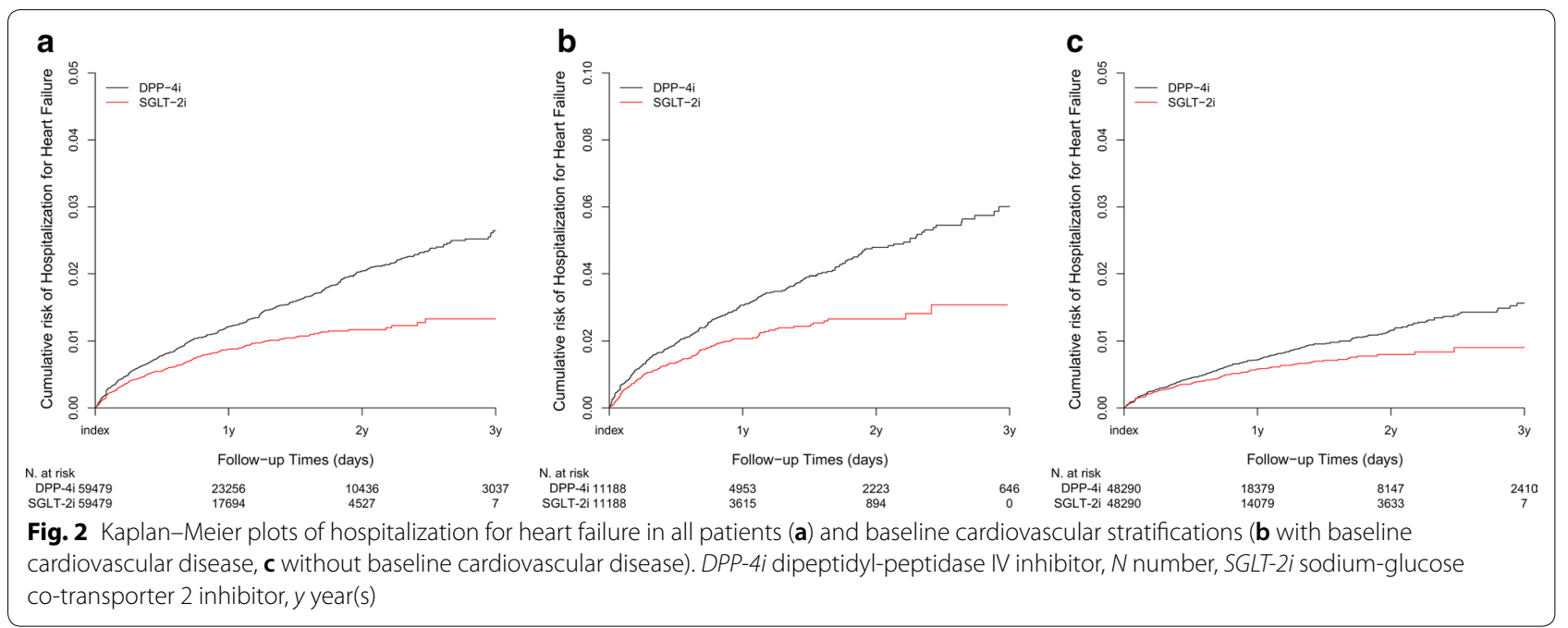

Table 2 The risk of hospitalization of SGLT-2i treated patients for heart failure according to follow-up period compared with DPP-4i treated patients

\begin{tabular}{|c|c|c|c|c|c|}
\hline $\begin{array}{l}\text { Days } \\
\text { after the drug } \\
\text { initiation }\end{array}$ & Events & HR & lower $\mathrm{Cl}$ & upper $\mathrm{Cl}$ & $p$ value \\
\hline \multicolumn{6}{|c|}{ Total patients $(\mathrm{N}=118,958)$} \\
\hline 30 days & 256 & 0.74 & 0.58 & 0.95 & 0.02 \\
\hline 90 days & 456 & 0.75 & 0.62 & 0.90 & 0.002 \\
\hline 180 days & 617 & 0.71 & 0.61 & 0.84 & $<0.001$ \\
\hline 1 year & 818 & 0.73 & 0.63 & 0.84 & $<0.001$ \\
\hline 3 years & 1025 & 0.66 & 0.58 & 0.75 & $<0.001$ \\
\hline \multicolumn{6}{|c|}{ Patients with underlying cardiovascular disease $(\mathrm{N}=22,376)$} \\
\hline 30 days & 117 & 0.62 & 0.43 & 0.91 & 0.01 \\
\hline 90 days & 220 & 0.73 & 0.5666 & 0.95 & 0.02 \\
\hline 180 days & 296 & 0.69 & 0.55 & 0.88 & $<0.001$ \\
\hline 1 year & 400 & 0.69 & 0.56 & 0.84 & $<0.001$ \\
\hline 3 years & 492 & 0.66 & 0.58 & 0.78 & $<0.001$ \\
\hline \multicolumn{6}{|c|}{ Patients without underlying cardiovascular disease $(N=96,580)$} \\
\hline 30 days & 123 & 1.09 & 0.76 & 1.55 & 0.65 \\
\hline 90 days & 218 & 0.89 & 0.68 & 1.16 & 0.39 \\
\hline 180 days & 298 & 0.83 & 0.66 & 1.05 & 0.12 \\
\hline 1 year & 403 & 0.83 & 0.68 & 0.84 & 0.06 \\
\hline 3 years & 502 & 0.76 & 0.63 & 0.95 & 0.003 \\
\hline
\end{tabular}

Cl 95\% confidence interval, $H R$ hazard ratio; $N$ number of patients

showed a significantly lower risk of hHF at 30,90 , and 180 days, as well as at 1 and 3 years after the initiating the drug vs. DPP-4i use (Table 2). In addition, among the patients with underlying CVD, SGLT-2i-treated patients were associated with a lower risk of hHF across all time points compared with DPP-4i. However, SGLT2i use only showed a lower risk of hHF with a significant difference 3 years after drug initiation compared with DPP-4i use among patients without underlying CVD.

\section{Discussion}

Our results show that SGLT-2i use reduced hHF compared with DPP-4i use. Patients with underlying CVD were associated with a reduced risk of hHF from 30 days after initiating the SGLT-2i to the third year, but patients without underlying CVD showed a significantly lower risk of $\mathrm{HF}$ in the third year.

In our study, SGLT-2i use reduced hHF by $36 \%$ compared with DPP-4i use, which is consistent with the results of the EMPA-REG OUTCOME trial, the CANVAS Program, and three observational studies $[6,8,9$, $14,15]$. In the EMPA-REG OUTCOME trial, empagliflozin reduced hHF by $35 \%$ (95\% CI, $0.50-0.85 ; \mathrm{p}=0.002)$ vs. placebo [6]. In addition, in the CANVAS Program, which included data integrated from the CANVAS and CANVAS-R trials, canagliflozin showed a 33\% reduction in hHF (95\% CI, 0.52-0.87) vs. placebo [8]. The finding that hHF began to be separated widely within 3 months and was maintained for 3 years in two previous RCTs has brought worldwide interest [2,16-18]. However, no statistical analysis has been performed to determine how soon patients benefit for hHF after using SGLT-2i; thus, there is a need to analyze the impact of hHF by dividing the duration of SGLT-2i use. Therefore, we analyzed hHF according to the duration of SGLT-2i use and showed that hHF was significantly lower in SGLT-2i new users vs. DPP-4i new users beginning 30 days after initiating SGLT-2i. This finding suggests that patients with T2DM and established CVD may benefit from HF management at the initiation of SGLT-2i use.

Several mechanisms have been proposed to explain the early HF protective effect of SGLT-2i. First, it induces a 
negative caloric balance and reduces hemoglobin A1c and body weight by causing glycosuria [16]. However, our results show that hHF decreased beginning 30 days after initiating the SGLT-2i, which was similar to previous RCTs $[6,8]$. The protective effect of HF is achievable within a short time although improvements in the glycemic and lipid profiles were modest at 3 months in those RCTs; thus, it likely occurs via a non-glycemic mechanism. Second, SGLT-2i improves hemodynamics through natriuresis, which was suggested as a pivotal mechanism to improve HF in the early phase of drug initiation in some studies [2,19-21]. Sodium glucose co-transporter 2 resorbs about $5 \%$ of the sodium under normal conditions and this capacity increases under a chronic hyperglycemic condition [2, 16, 22-24]. Thus, SGLT-2i decrease plasma volume which consequently reduces preload and ventricular filling pressure, and lowers blood pressure, which causes a reduction in afterload and improves subendocardial blood flow. Third, a shift in fuel energetics has been suggested as a protective mechanism for HF and has been called the "thrifty substrate" hypothesis $[17,25]$. This hypothesis proposes that SGLT-2i induce ketogenesis, including $\beta$-hydroxybutyrate, in patients with T2DM $[25,26]$. $\beta$-hydroxybutyrate is freely taken up by the heart and used as an energy-efficient "superfuel" to improve cardiac metabolism, such as oxygen consumption, at the mitochondrial level and also increase cardiac hydraulic efficiency in animal HF model $[17,18,27,28]$. Finally, SGLT-2i may exert a cardioprotective effect by lowering uric acid level. Plasma uric acid level is associated with congestive HF and CVD [29-31]. SGLT-2i use causes secretion of uric acid via the GLUT9 transporter, resulting in a $10-15 \%$ reduction in plasma uric acid level $[32,33]$.

Previous RCTs included only patients with underlying CVD or patients at high risk for CVD; thus, there is a concern whether SGLT-2i have similar benefits in patients with T2DM who do not have established CVD. One observational study showed that SGLT-2i use on T2DM patients without underlying DM complication showed lower HR without statistical significance (adjusted HR $=0.83 ; 95 \% \mathrm{CI}=0.54-1.27 ; \mathrm{p}=0.40$ ) [34]. However, in our study, hHF decreased significantly in the third year among patients without underlying CVD. The difference between our study result and that of Gautam et al. was probably due to the fact that we performed subgroup analysis according to the presence of underlying cardiovascular disease, whereas their analysis was based on the presence of any DM complications (any microvascular or macrovascular complications). Furthermore, they enrolled just 5000 patients, which was smaller than our study. To our knowledge, no observational study has analyzed the HF protective effect of SGLT-2i use with
CVD stratification. This is the first study to estimate the HF protective effect among patients without established CVD.

In our study, SGLT-2i use reduced hHF compared with DPP-4i use beginning 30 days after initiating the drug among patients with underlying CVD, which was far earlier than patients without CVD. This result is in agreement with the results of a previous EMPA-REG sub study showing that SGLT-2i use was associated with a lower risk of hospitalization for HF from 360 to 1440 days after the drug initiation [35]. Also, although there was no statistical analysis to determine whether HF risk varies with follow-up period in the CANVAS program, early separation in the $\mathrm{K}-\mathrm{M}$ curve of hHF after drug initiation was more prominent in the EMPA-REG OUTCOME trial, where all participants had previous history of CVD, compared to the CANVAS program that enrolled $34.4 \%$ participants who had no previous history of CVD $[6,8]$. Our finding and the results from previous studies suggests that the effectiveness of HF protective mechanism with SGLT-2i use could be different depending on the patient's underlying CVD. As our results and results of previous RCTs show, patients with T2DM and established CVD could benefit from SGLT-2i use beginning when the drug is initiated. Among the several mechanisms described above, such as a hemodynamic effect, and shift in fuel energetics, SGLT-2i could be more beneficial to patients with established CVD. One study showed that SGLT-2i decreased systolic blood pressure and increased diuresis among T2DM patients in just $48 \mathrm{~h}$ after initiation [20], and that this effect lasts relatively longer [22]. Also, after just a single dose of SGLT-2i, T2DM patients showed increased ketogenesis, which could directly benefit hemodynamics in heart failure patients [17, 25, 28]. Maybe these hemodynamic or metabolic changes could protect T2DM patients with established CVD immediately after SGLT-2i use, but later in patients without established CVD. However, our study did not analyze laboratory or echocardiographic results to determine the underlying mechanisms. Therefore, further evaluation is needed to identify the HF protective mechanism in patients with or without underlying CVD.

Several strengths of the present study should be mentioned. First, to our knowledge, this is the first study to estimate HF risk with CVD stratification and show that the HF protective effect of SGLT-2i can vary depending on whether patients have established CVD or not. Second, our results are the first to show that SGLT-2i have significant effects on HF beginning just 30 days after initiating the drug. This could be a meaningful finding and confirm the conjectures of previous studies. Third, this study was a nationwide population-based cohort study including over 50,000 new users of SGLT-2i. Finally, 
our study compared the SGLT-2i class, which is of great interest, with DPP-4i, one of the most widely used oral hypoglycemic agents in the world. By comparing these two drug classes and strictly adjusting all other oral hypoglycemic agents associated with hHF, physicians who consider prescribing both drugs will be directly helped in clinical situations with real world data.

Our research had some limitations. First, we used claims data, which do not contain information about measurements such as laboratory or echocardiography results, New York Heart Association Functional Classification of heart failure, socio-economic status, or diabetes duration; consequently, residual confounding factors probably existed. To compensate for this limitation, we performed propensity score matching with 55 variables including several complications of diabetes and HF medications. However, propensity score matching is not a remedy for all confounding factors, so our results should be interpreted with caution. In addition, we only adjusted prescribed medication before, but not after, the index date and, thus, there are still residual confounding factors. Second, we could not analyze mortality after use of SGLT-2i because the HIRA database does not contain mortality data. As previous RCTs and observational studies have already shown that SGLT-2i use is associated with improved mortality, our study could be more meaningful as it focused on the effect of SGLT-2i on HF in greater detail. Third, we could not analyze the HF risk of SGLT-2i according to the presence of underlying HF because there were only two thousand patients with underlying heart failure, which was too small for analysis.

\section{Conclusions}

Our results suggest that SGLT-2i reduced hHF compared with DPP-4i. A HF protective effect of SGLT-2i vs. DPP$4 \mathrm{i}$ was shown 30 days after initiating the SGLT-2i among patients with established CVD, but this effect appeared later in patients without established CVD.

\section{Additional file}

Additional file 1: Table S1. Baseline characteristics of all patients before propensity score matching. Data presented as frequencies in percentage or means (SD). *Confirmed by diagnosis code (International Classification of Diseases, 10th revision). ACEl angiotensin-converting-enzyme inhibitor, AMI acute myocardial infarction, $A R B$ angiotensin II receptor antagonists, DPP-4i dipeptidyl-peptidase IV inhibitor, NOAC novel oral anticoagulant, SD standard deviation, SGLT2i sodium-glucose co-transporter 2 inhibitor, SU sulfonylurea. Table S2. Baseline characteristics of matched patients with established cardiovascular disease. Data presented as frequencies in percentage or means (SD). Less than 0.1 (10\%) on the absolute value of standardized difference was considered as a negligible difference between groups. The mean (SD) standardized difference of all covariates was 1.01\% (1.33\%). ${ }^{*}$ Confirmed by diagnosis code (International Classification of Diseases, 10th revision). ACEl angiotensin-converting-enzyme inhibitor, $A M I$ acute myocardial infarction, $A R B$ angiotensin II receptor antagonists, DPP-4i dipeptidyl-peptidase IV inhibitor, NOAC novel oral anticoagulant, SD standard deviation, SGLT2i sodium-glucose co-transporter 2 inhibitor, SU sulfonylurea. Table S3. Baseline characteristics of matched patients without established cardiovascular disease. Data presented as frequencies in percentage or means (SD). Less than 0.1 (10\%) on the absolute value of standardized difference was considered as a negligible difference between groups. The mean (SD) standardized difference of all covariates was $0.96 \%$ (2.03\%). *Confirmed by diagnosis code (International Classification of Diseases, 10th revision). ACEl angiotensin-convertingenzyme inhibitor, $A R B$ angiotensin II receptor antagonists, DPP-4i dipeptidyl-peptidase IV inhibitor, NOAC novel oral anticoagulant, SD standard deviation, SGLT2i sodium-glucose co-transporter 2 inhibitor, SU sulfonylurea. The English in this document has been checked by at least two professional editors, both native speakers of English. For a certificate, please see: http://textcheck.com/certificate/index/dlr6 Nw.

\section{Abbreviations}

CANVAS: Canagliflozin Cardiovascular Assessment Study Program; Cl: confidence interval; CVD: cardiovascular disease; DM: diabetes mellitus; DPP-4i: dipeptidyl peptidase-4 inhibitors; EMPA-REG OUTCOME: Empagliflozin Cardiovascular Outcome Event Trial in Type 2 Diabetes Mellitus Patients trial; HF: heart failure; hHF: hospitalization for heart failure; HR: hazard ratio; K-M: Kaplan-Meier; N: number; RCT: randomized controlled trial; SD: standard deviation; SGLT-2i: sodium glucose co-transporter 2 inhibitors; T2DM: type 2 diabetes mellitus.

\section{Authors' contributions}

YGK designed the study, analyzed data, and wrote the manuscript. SJH, DJK, and KWL contributed to the discussion and reviewed and edited the manuscript. HJK designed the study, analyzed data, and reviewed the manuscript as a corresponding author. All authors qualify for authorship according to International Committee of Medical Journal Editors criteria and gave final approval for publication. All authors read and approved the final manuscript.

\section{Author details}

${ }^{1}$ Department of Medical Sciences, Ajou University Graduate School, Suwon, Republic of Korea. ${ }^{2}$ Department of Internal Medicine, Incheon Medical Center, Incheon, Republic of Korea. ${ }^{3}$ Department of Endocrinology and Metabolism, Ajou University School of Medicine, Suwon, Republic of Korea.

\section{Acknowledgements}

This study utilized data from the Korean Health Insurance Review and Assessment Service, and the results are not affected by the opinion of the Korean Health Insurance Review and Assessment Service.

\section{Competing interests}

The authors declare that they have no competing interests.

\section{Availability of data and materials}

The datasets analyzed in this study are available from the database of Korean Health Insurance Review and Assessment Service.

\section{Consent for publication}

Not applicable.

\section{Ethics approval and consent to participate}

All participants gave informed consent; the study was approved by the local ethics committee and is in accordance with the Declaration of Helsinki.

\section{Funding}

This research was supported by Basic Science Research Program through the National Research Foundation of Korea (NRF) funded by the Ministry of Education (NRF-2017R1D1A1B03029340).

\section{Publisher's Note}

Springer Nature remains neutral with regard to jurisdictional claims in published maps and institutional affiliations. 
Received: 7 April 2018 Accepted: 20 June 2018

Published online: 23 June 2018

\section{References}

1. Metra M, Carubelli V, Ravera A, Stewart Coats AJ. Heart failure 2016: still more questions than answers. Int J Cardiol. 2017;227:766-77.

2. Lytvyn Y, Bjornstad P, Udell JA, Lovshin JA, Cherney DZI. Sodium glucose cotransporter-2 inhibition in heart failure: potential mechanisms, clinical applications, and summary of clinical trials. Circulation. 2017;136(17):1643-58.

3. Bell DS. Heart failure: the frequent, forgotten, and often fatal complication of diabetes. Diabetes Care. 2003;26(8):2433-41.

4. Home PD, Pocock SJ, Beck-Nielsen H, Gomis R, Hanefeld M, Jones NP, Komajda M, McMurray JJ. Rosiglitazone evaluated for cardiovascular outcomes-an interim analysis. N Engl J Med. 2007;357(1):28-38.

5. Scirica BM, Bhatt DL, Braunwald E, Steg PG, Davidson J, Hirshberg B, Ohman P, Frederich R, Wiviott SD, Hoffman EB, et al. Saxagliptin and cardiovascular outcomes in patients with type 2 diabetes mellitus. N Engl J Med. 2013;369(14):1317-26.

6. Zinman B, Wanner C, Lachin JM, Fitchett D, Bluhmki E, Hantel S, Mattheus M, Devins T, Johansen OE, Woerle HJ, et al. Empagliflozin, cardiovascular outcomes, and mortality in type 2 diabetes. N Engl I Med. 2015;373(22):2117-28

7. Kaku K, Lee J, Mattheus M, Kaspers S, George J, Woerle HJ. Empagliflozin and cardiovascular outcomes in asian patients with type 2 diabetes and established cardiovascular disease-results from EMPA-REG OUTCOME((R)). Circ J. 2017;81(2):227-34.

8. Neal B, Perkovic V, Mahaffey KW, de Zeeuw D, Fulcher G, Erondu N, Shaw W, Law G, Desai M, Matthews DR. Canagliflozin and cardiovascular and renal events in type 2 diabetes. N Engl J Med. 2017;377(7):644-57.

9. Persson F, Nystrom T, Jorgensen ME, Carstensen B, Gulseth HL, Thuresson M, Fenici P, Nathanson D, Eriksson JW, Norhammar A, et al. Dapagliflozin is associated with lower risk of cardiovascular events and all-cause mortality in people with type 2 diabetes (CVD-REAL Nordic) when compared with dipeptidyl peptidase-4 inhibitor therapy: a multinational observational study. Diabetes Obes Metab. 2018;20(2):344-51.

10. Zannad F, Cannon CP, Cushman WC, Bakris GL, Menon V, Perez AT, Fleck PR, Mehta CR, Kupfer S, Wilson C, et al. Heart failure and mortality outcomes in patients with type 2 diabetes taking alogliptin versus placebo in EXAMINE: a multicentre, randomised, double-blind trial. Lancet. 2015;385(9982):2067-76.

11. Green JB, Bethel MA, Armstrong PW, Buse JB, Engel SS, Garg J, Josse R, Kaufman KD, Koglin J, Korn S, et al. Effect of sitagliptin on cardiovascular outcomes in type 2 diabetes. N Engl J Med. 2015:373(3):232-42.

12. Kim YG, Yoon D, Park S, Han SJ, Kim DJ, Lee KW, Park RW, Kim HJ. Dipeptidyl peptidase-4 inhibitors and risk of heart failure in patients with type 2 diabetes mellitus: a population-based cohort study. Circ Heart Fail. 2017;10(9):e003957.

13. Filion KB, Azoulay L, Platt RW, Dahl M, Dormuth CR, Clemens KK, Hu N, Paterson JM, Targownik L, Turin TC, et al. A multicenter observational study of incretin-based drugs and heart failure. N Engl J Med. 2016;374(12):1145-54.

14. Kosiborod M, Cavender MA, Fu AZ, Wilding JP, Khunti K, Holl RW, Norhammar A, Birkeland KI, Jorgensen ME, Thuresson M, et al. Lower risk of heart failure and death in patients initiated on sodium-glucose cotransporter-2 inhibitors versus other glucose-lowering drugs: The CVD-REAL Study (comparative effectiveness of cardiovascular outcomes in new users of sodium-glucose cotransporter-2 inhibitors). Circulation. 2017:136(3):249-59.

15. Nystrom T, Bodegard J, Nathanson D, Thuresson M, Norhammar A, Eriksson JW. Novel oral glucose-lowering drugs are associated with lower risk of all-cause mortality, cardiovascular events and severe hypoglycaemia compared with insulin in patients with type 2 diabetes. Diabetes Obes Metab. 2017:19(6):831-41.

16. Heerspink HJ, Perkins BA, Fitchett DH, Husain M, Cherney DZ. Sodium glucose cotransporter 2 inhibitors in the treatment of diabetes mellitus: cardiovascular and kidney effects, potential mechanisms, and clinical applications. Circulation. 2016;134(10):752-72.
17. Ferrannini E, Mark M, Mayoux E. CV protection in the EMPA-REG OUTCOME trial: a "Thrifty Substrate" hypothesis. Diabetes Care. 2016:39(7):1108-14

18. Mudaliar S, Alloju S, Henry RR. Can a shift in fuel energetics explain the beneficial cardiorenal outcomes in the EMPA-REG OUTCOME study? A Unifying Hypothesis. Diabetes care. 2016;39(7):1115-22.

19. Tanaka A, Node K. Emerging roles of sodium-glucose cotransporter 2 inhibitors in cardiology. J Cardiol. 2017;69(3):501-7.

20. Solini A, Giannini L, Seghieri M, Vitolo E, Taddei S, Ghiadoni L, Bruno RM. Dapagliflozin acutely improves endothelial dysfunction, reduces aortic stiffness and renal resistive index in type 2 diabetic patients: a pilot study. Cardiovasc Diabetol. 2017;16(1):138

21. Habibi J, Aroor AR, Sowers JR, Jia G, Hayden MR, Garro M, Barron B, Mayoux E, Rector RS, Whaley-Connell A, et al. Sodium glucose transporter 2 (SGLT2) inhibition with empagliflozin improves cardiac diastolic function in a female rodent model of diabetes. Cardiovasc Diabetol. 2017;16(1):9.

22. Baker WL, Smyth LR, Riche DM, Bourret EM, Chamberlin KW, White WB. Effects of sodium-glucose co-transporter 2 inhibitors on blood pressure: a systematic review and meta-analysis. J Am Soc Hypertens (JASH). 2014:8(4):262-75.

23. Tanaka H, Takano K, lijima H, Kubo H, Maruyama N, Hashimoto T, Arakawa K, Togo M, Inagaki N, Kaku K. Factors affecting canagliflozin-induced transient urine volume increase in patients with type 2 diabetes mellitus. Adv Ther. 2017;34(2):436-51.

24. Wilding JP, Norwood P, T'Joen C, Bastien A, List JF, Fiedorek FT. A study of dapagliflozin in patients with type 2 diabetes receiving high doses of insulin plus insulin sensitizers: applicability of a novel insulin-independent treatment. Diabetes Care. 2009;32(9):1656-62.

25. Ferrannini E, Baldi S, Frascerra S, Astiarraga B, Heise T, Bizzotto R, Mari A, Pieber TR, Muscelli E. Shift to fatty substrate utilization in response to sodium-glucose cotransporter 2 inhibition in subjects without diabetes and patients with type 2 diabetes. Diabetes. 2016;65(5):1190-5.

26. Daniele G, Xiong J, Solis-Herrera C, Merovci A, Eldor R, Tripathy D, DeFronzo RA, Norton L, Abdul-Ghani M. Dapagliflozin enhances fat oxidation and ketone production in patients with type 2 diabetes. Diabetes Care. 2016;39(11):2036-41.

27. Veech RL. The therapeutic implications of ketone bodies: the effects of ketone bodies in pathological conditions: ketosis, ketogenic diet, redox states, insulin resistance, and mitochondrial metabolism. Prostaglandins Leukot Essent Fatty Acids. 2004:70(3):309-19.

28. Kolwicz SC Jr, Airhart S, Tian R. Ketones step to the plate: a game changer for metabolic remodeling in heart failure? Circulation. 2016;133(8):689-91.

29. Huang H, Huang B, Li Y, Huang Y, Li J, Yao H, Jing X, Chen J, Wang J. Uric acid and risk of heart failure: a systematic review and meta-analysis. Eur J Heart Fail. 2014;16(1):15-24.

30. Lytvyn Y, Perkins BA, Cherney DZ. Uric acid as a biomarker and a therapeutic target in diabetes. Can J Diabetes. 2015;39(3):239-46.

31. Ndrepepa G, Braun S, King L, Hadamitzky M, Haase HU, Birkmeier KA, Schomig A, Kastrati A. Association of uric acid with mortality in patients with stable coronary artery disease. Metab Clin Exp. 2012;61(12):1780-6.

32. Lytvyn Y, Skrtic M, Yang GK, Yip PM, Perkins BA, Cherney DZ. Glycosuriamediated urinary uric acid excretion in patients with uncomplicated type 1 diabetes mellitus. Am J Physiol Renal Physiol. 2015;308(2):F77-83.

33. Ferrannini E, Solini A. SGLT2 inhibition in diabetes mellitus: rationale and clinical prospects. Nat Rev Endocrinol. 2012;8(8):495-502.

34. Gautam S, Agiro A, Barron J, Power T, Weisman H, White J. Heart failure hospitalization risk associated with use of two classes of oral antidiabetic medications: an observational, real-world analysis. Cardiovasc Diabetol. 2017;16(1):93.

35. Fitchett D, Butler J, van de Borne P, Zinman B, Lachin JM, Wanner C, Woerle HJ, Hantel S, George JT, Johansen OE, et al. Effects of empagliflozin on risk for cardiovascular death and heart failure hospitalization across the spectrum of heart failure risk in the EMPA-REG OUTCOME(R) trial. Eur Heart J. 2018;39(5):363-70. 